\title{
Research Paper: Validity and Reliability of Family Time and Routines Index in the Families of Children With Autism
}

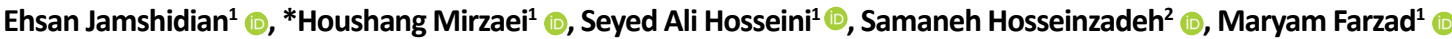

1. Department Of Occupational Therapy, University of Social Welfare and Rehabilitation Sciences, Tehran, Iran. 2. Department of Biostatistics, University of Social Welfare and Rehabilitation Sciences, Tehran, Iran.

\begin{tabular}{|c|c|}
\hline $\begin{array}{l}\text { Use your device to scan } \\
\text { and read the article online }\end{array}$ & ditation Jamshidian E, Mirzaei H, Hosseini SA, Hosseinzadeh S, Farzad M. [Validity and Reliability of Family Time and \\
\hline 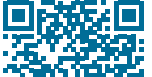 & $\begin{array}{l}\text { Routines Index in the Families of Children With Autism (Persian)]. Archives of Rehabilitation. 2019; 20(2):158-173. http://dx.doi. } \\
\text { org/10.32598/rj.20.2.158 }\end{array}$ \\
\hline 13p4 & d. http://dx.doi.org/10.32598/rj.20.2.158 \\
\hline
\end{tabular}

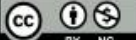

Received: 17 Feb 2019

Accepted: 13 May 2019

Available Online: $01 \mathrm{Jul} 2019$

\begin{abstract}
Objective Autism spectrum disorders are among the most prevalent developmental disorders. Diagnostic criteria for these disorders consist of the two general categories of symptoms, including deficiencies in communication and social interactions, and limited, repetitive and stereotypic patterns in behavior, interests, and activities. Routines are well-established and the regular patterns of occupations and activities that provide structure and stability for everyday life and add meaning to it can affect health. The disabling nature of autism and its numerous associated problems unable families to develop effective and meaningful routines; consequently, the integrity and solidarity of family are lost and family is disturbed. A proper tool is required to evaluate everyday routine in the families of children with autism. The present study investigated the validity and reliability of the Family Time and Routines Index (FTRI) in the Iranian families of children with autism.

Materials \& Methods This was a non-experimental methodological study. The statistical population of the study consisted of all children with autism in Tehran City, Iran. Among them, 100 parents of children with autism referring to autism centers and private clinics in Tehran were selected by the convenience sampling method; they were entered into the study, according to the inclusion criteria. Inclusion criteria were the diagnosis of autism by a child psychiatrist, based on the Diagnostic and Statistical Manual of Mental Disorders, Fifth Edition (DSM-V), the age of 3-11 years, and the absence of blindness, deafness, epilepsy, and other disabilities in the child. Moreover, the subjects with no desire to cooperate or uncompleted the questionnaires were excluded from the study. The required data were collected using demographic information questionnaire and FTRI completed by parents. After obtaining the permission of the designer of the questionnaire for translation and the investigation of its psychometric properties, the questionnaire was translated according to the International Quality of Life Assessment (IQOLA) project. The face validity and content validity of the questionnaire were evaluated using experts and parents' opinions; then, a preliminary test was conducted in which the questionnaire was completed by 10 parents of children with autism to determine the appropriate time to complete the questionnaire. Consequently, 100 parents of children with autism completed the demographic information questionnaire and FTRI. Furthermore, to determine the test-retest reliability, after 2 weeks, 33 parents of children with autism re-completed the questionnaire. To examine the face validity, item impact score was calculated. To assess the content validity, Content Validity Ratio (CVR) and Content Validity Index (CVI) was used. Internal Consistency Coefficient (ICC) was used for assessing the test-retest reliability of the
\end{abstract}

\section{* Corresponding Author:}

Houshang Mirzaei, PhD.

Address: Department Of Occupational Therapy, University of Social Welfare and Rehabilitation Sciences, Tehran, Iran.

Tel: +98 (936) 3235543

E-Mail: jamshidian.ot@gmail.com 
Keywords:

Autism spectrum disorder, Family, Occupational therapy, Reproducibility of results, Validation studies scale. Moreover, the internal consistency of the whole questionnaire and its subscales was assessed by Cronbach's alpha coefficient.

Results Item impact score for all items of the questionnaire was over 1.5. The CVR for each item of the questionnaire was between 0.8 and 1 and for the whole questionnaire was equal to 0.87 . The CVI for the items of the questionnaire was between 0.83 and 1 and for the whole questionnaire was equal to 0.97 . Additionally, the intraclass correlation coefficient for the total score was equal to 0.96 and Cronbach's alpha coefficient was calculated as 0.88 . Moreover, the lowest Cronbach's alpha coefficient related to the subscale of child routines $(0.70)$ and the highest related to the subscale relative's connection routines (0.86).

Conclusion The obtained results indicated that FTRI has acceptable validity and reliability in the Iranian samples and can be a useful research and clinical tool for assessing the family routines. 
This Page Intentionally Left Blank 


\title{
بررسى روايى و يايايى ثرسشنامه زمان و روالهاى رايج روزمره خانواده (FTRI) در خانوادههاى كودكان داراي اتُيسم
}

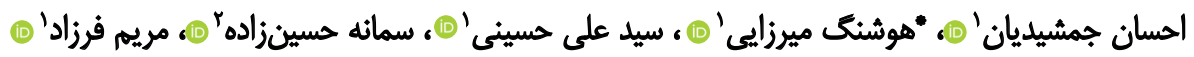 \\ 1 - كروه كاردرمانى، دانشكاه علوم بهزيستى و توانبخشى، تهران، ايران. \\ r- كروه آمار زيستى، دانشكاه علوم بهزيستى و توانبخشى، تهران، ايران.
}

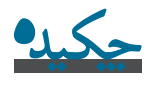

هدف اختلالات طيف أتيسم از شايعترين اختلالات رشدى است. معيارهاى تشخيصى اين دسته از اختلالات دو دسته كلى علائم شامل نقص در

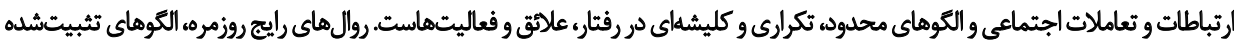

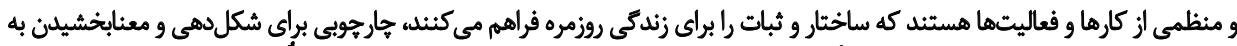

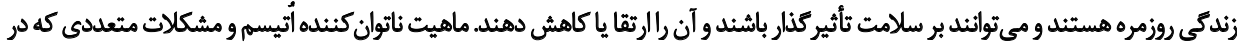

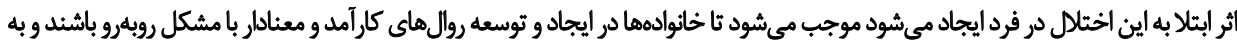

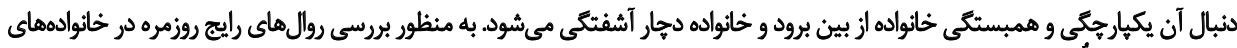

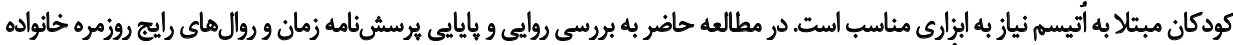

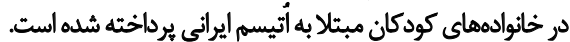

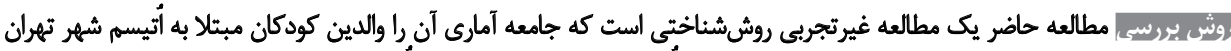

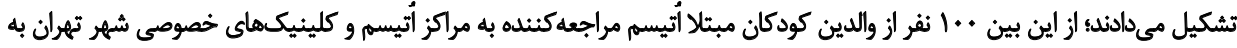

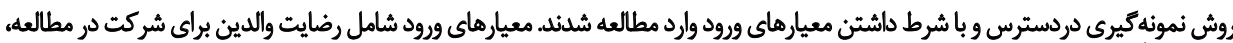

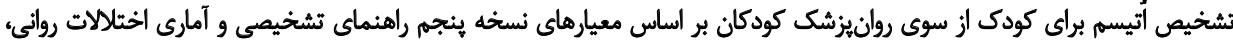

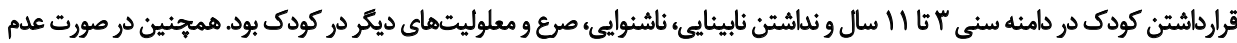

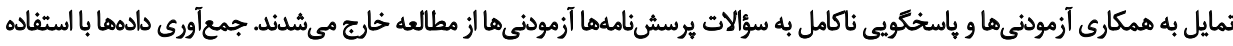

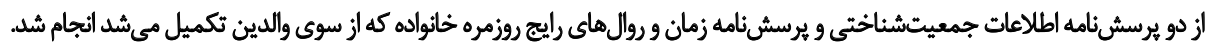

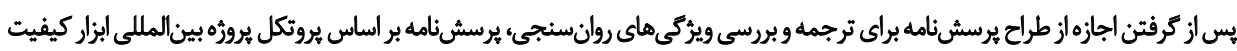

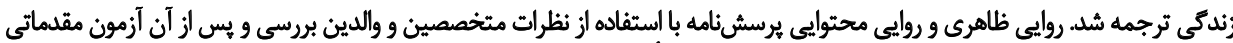

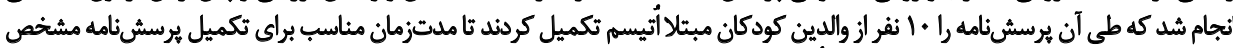

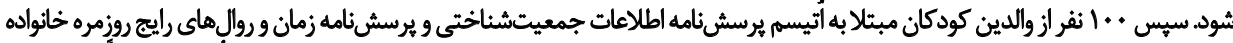

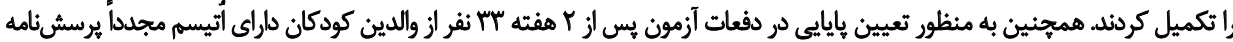

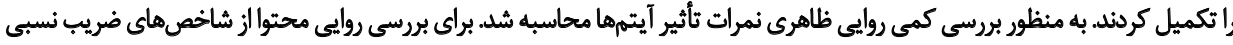

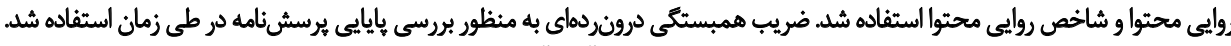

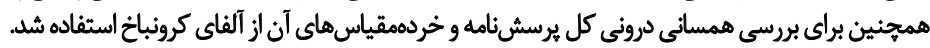

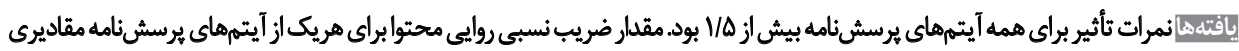

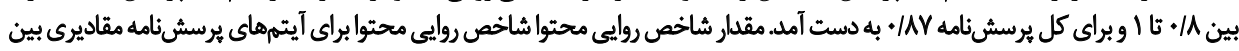

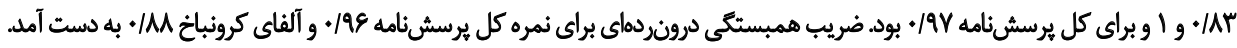

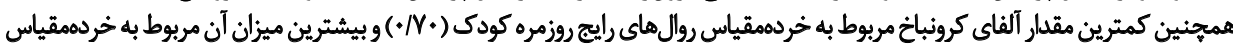

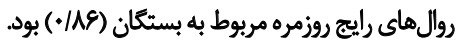

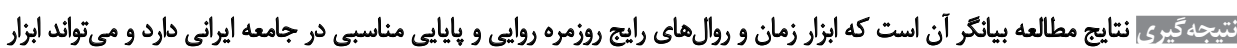

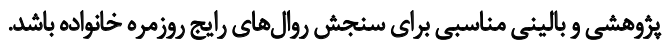

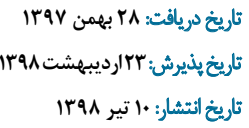

كليدوأوها:

اخحتلال طيف أتيسهم،

تكراريذيرى نتايج،

خانواده، كاردرماني، مطالعات اعتبارسنجي كاردماني، 


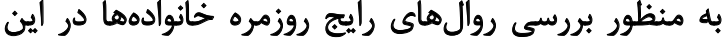

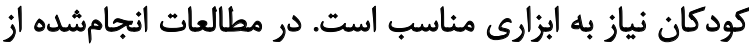

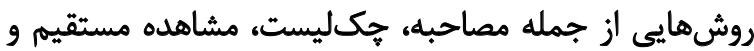

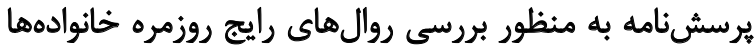

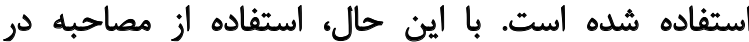

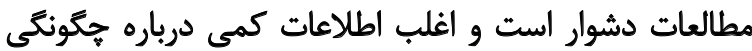

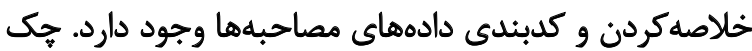

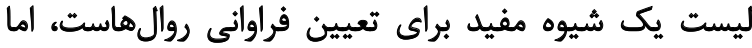

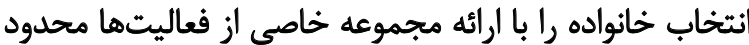

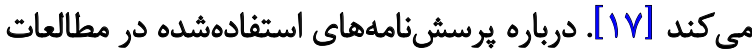

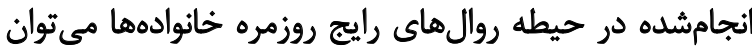

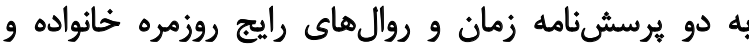
يرسشنامه روالهائ رايج روزمره خانواده اشاره كرد. يرسشنامه زمان و روالهاى رايج روزمره خانواده كه نسخه

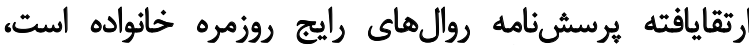

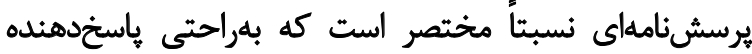

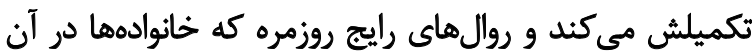

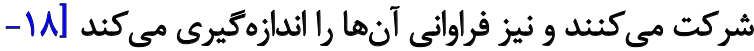

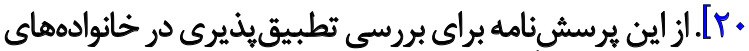

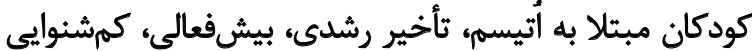

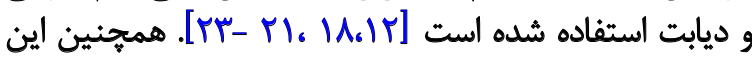

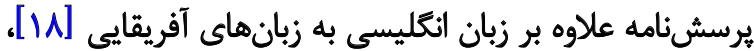

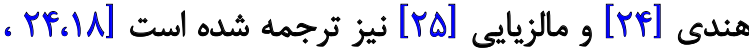

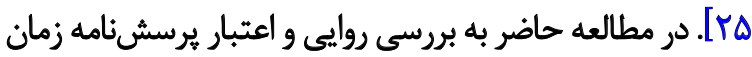

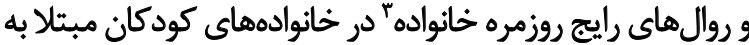
أتيسم ايرانى يرداخته شده است.

$$
\text { روش بروشى }
$$

\section{مطالعه حاضر مطالعهاى غيرتجربى و روششناختى است}

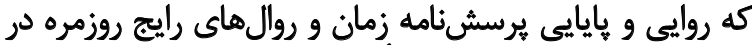

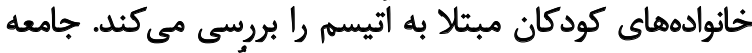

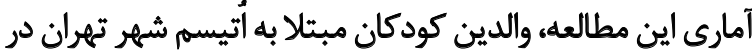

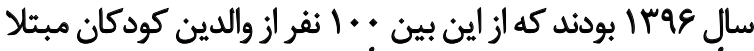

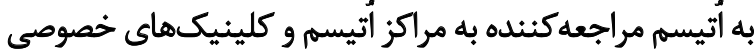

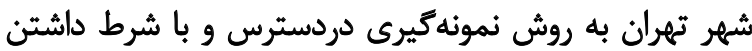

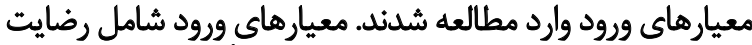

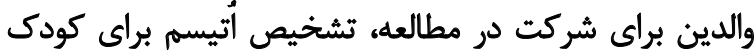

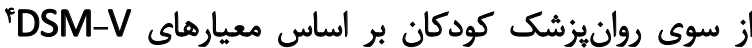

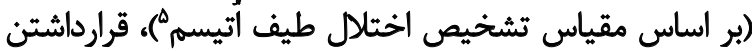

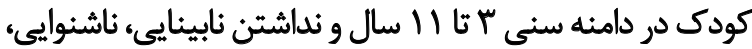

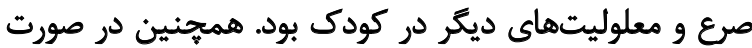

3. Family Time And Routines Index (FTRI)

4. Diagnostic and Statistical Manual of Mental Disorders, $5^{\text {th }}$ Edition 5. Autism Spectrum Disorders Diagnosis Scale (ASDDS)
مقلمه

اختلالات طيف أتيسم از شايعترين اختلالات رشدى است بتري

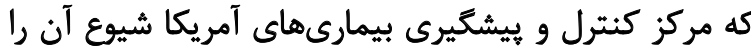

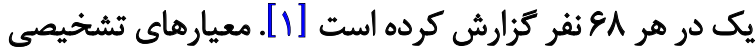

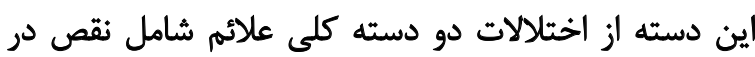

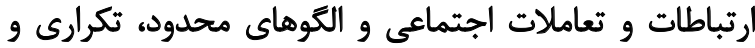

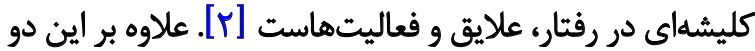

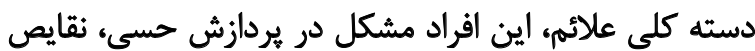

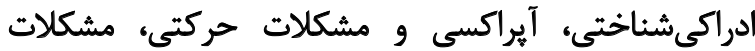

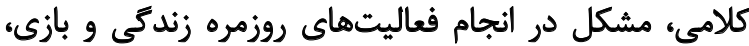

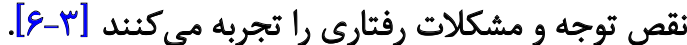
اين مشكلات مىتواند بر تعداد، نوع و كيفيت فعاليتهاى

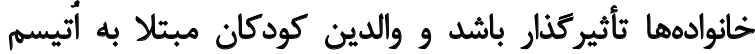

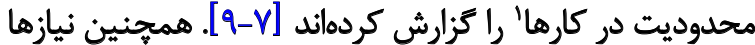

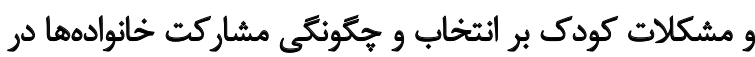

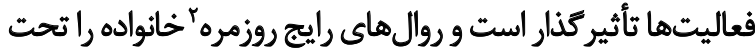
تأثير قرار مىدهد [-1]]

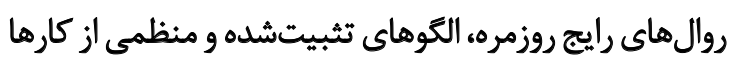

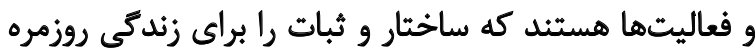

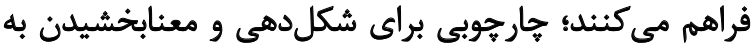

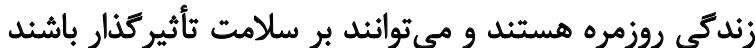

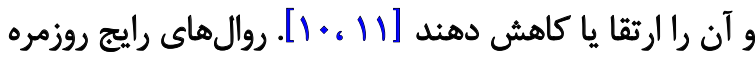

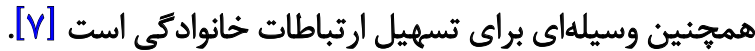

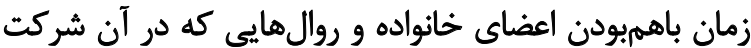

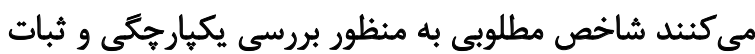

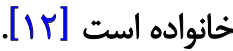
روالها يايهاي محكم را براي حمايت بينفردى در خانواده

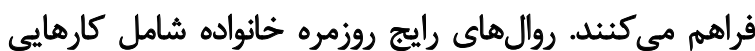

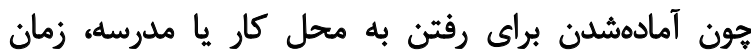

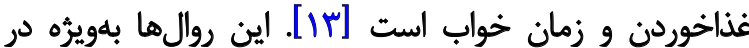

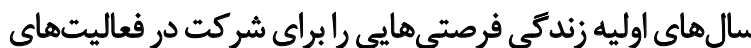

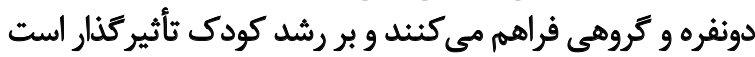

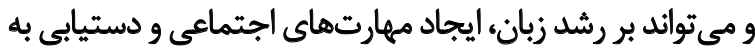
مهارتهاى تحصيلى در آينده تأثيركذار باشد [1F]

ماهيت ناتوان كنيده أتيسمو ومشكلات متعددى كه در اثر ابتلابه

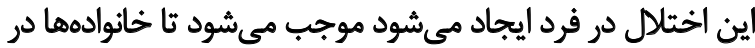

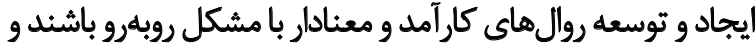

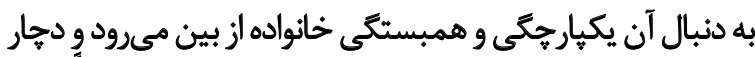

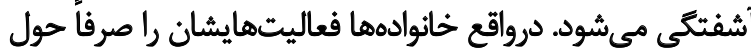

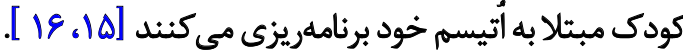

1. Occupations

2. Routines 
عبارات ياوجود نارسايى در معانى كلمات انجام شد. براي بررسى

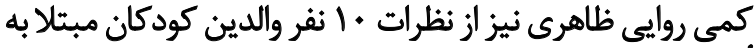

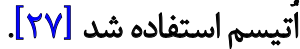

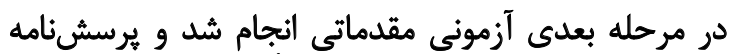

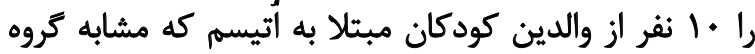

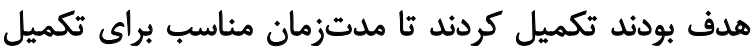

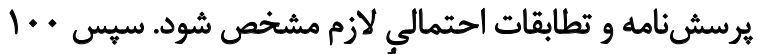

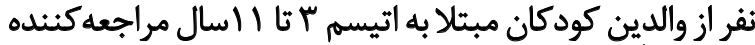

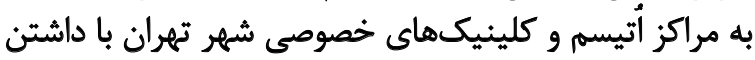

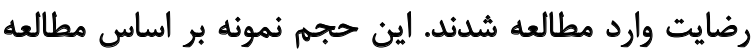

$$
\text { مقدماتى و با فرمول زير تعيين شد. }
$$

$$
n=\frac{\frac{z_{a}^{2}}{2} \times s^{2}}{d^{2}}
$$

\section{$(Z=1 / 96 ، d=0 / 05 ، S=0 / 25)$}

ابتدارضايت والدين كرفته شدو سيس توضيحات لازم در ارتباط

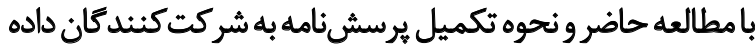

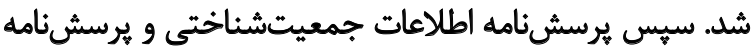

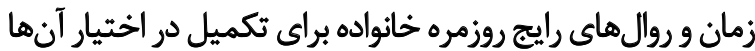

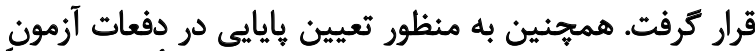

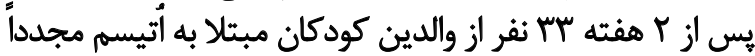

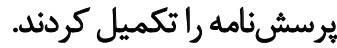

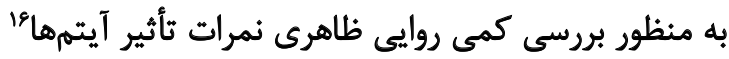

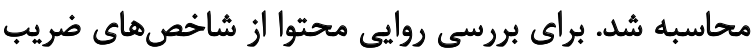

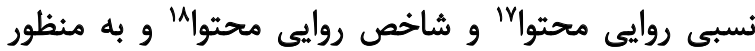

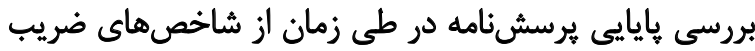

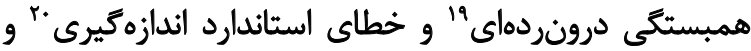

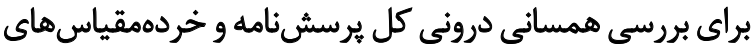
آن از آلفاى كرونباخ استفاده شد.

ياقتهها

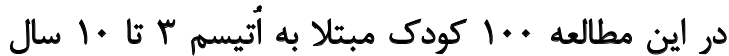

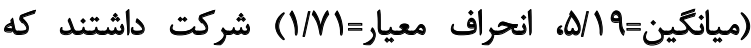

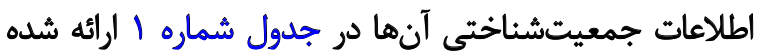

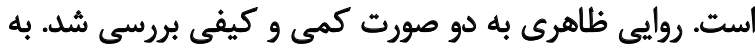

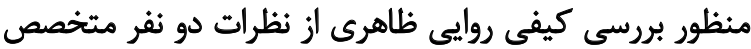

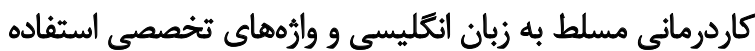

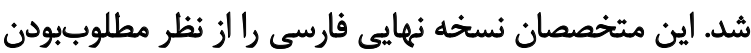

16. Item impact scores

17. Content Validity Ratio (CVR)

18. Content Validity Index (CVI)

19. Interclass Correlation Coefficient (ICC)

20. Standard Error of Measure (SEM)

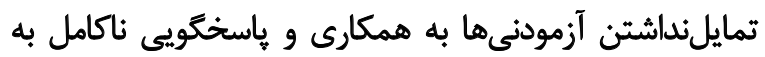

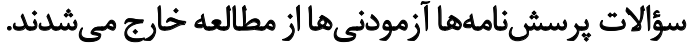
جمعآورى دادها با استفاده از دو يرسشنامه اطلاعات

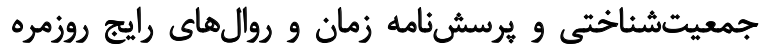

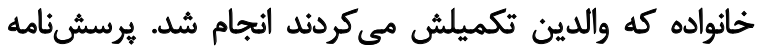

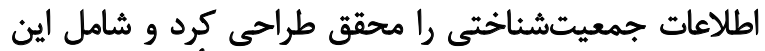
موارد بود: جنسيت كودك، سن كودك و سطح أتيسه.

يرسشنامه زمان و روالهاى رايج روزمره خانواده را مك روز روزي

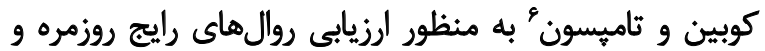

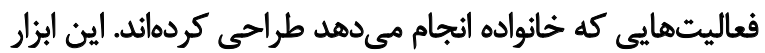

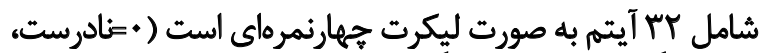

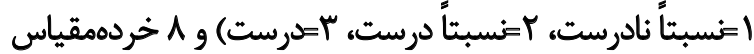

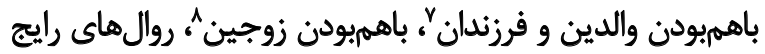

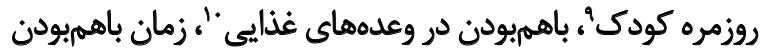

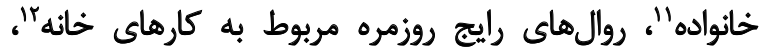

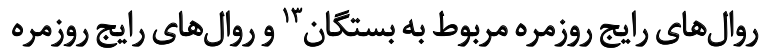

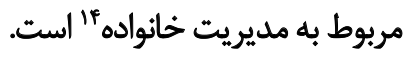

از جمع نمرات خام خردهمقياسها نمره كل يرسش نامنامه زمان

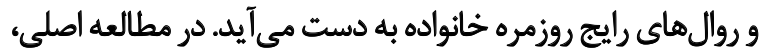

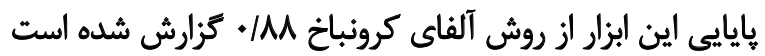

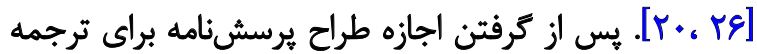

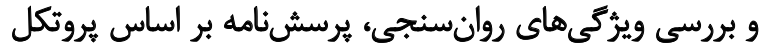

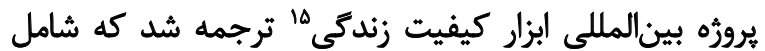

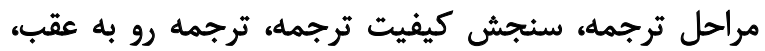

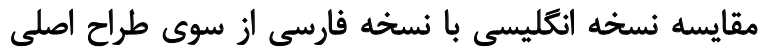

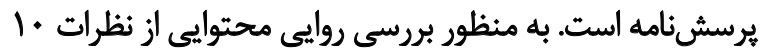

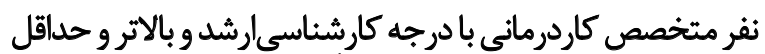

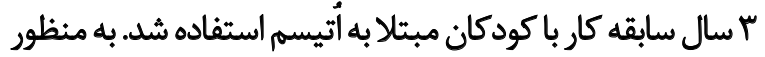

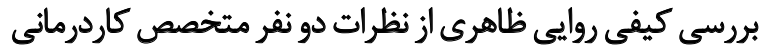

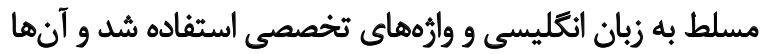

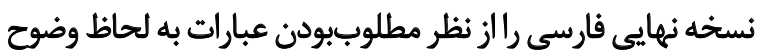
و كاربرد زبان مشترك بررسى كردند.

همجنين، براى دريافت نظرات زروه هدف، مصاحبهاى با • انفر

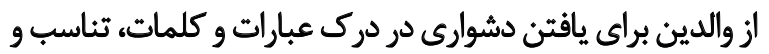

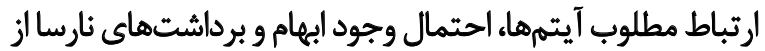

6. McCubbin \& Thompson

7. Parent-child togetherness

8. Couple togetherness

9. Child routines

10. Meal's together subscale

11. Family time together

12. Family chores routines

13. Relatives connection routines

14. Family management routines

15. International Quality of Life Assessment Project (IQOLA) 
جدول (. اطلاعات جمعيتششناختي

\begin{tabular}{|c|c|c|c|}
\hline \multicolumn{2}{|c|}{ درصد فراواثى } & \multirow{2}{*}{\multicolumn{2}{|c|}{ متغير }} \\
\hline بازأزمون (تعداد=سrr) & آزمون (تعداد=+ + 1) & & \\
\hline 19 & $M$ & يّر & \\
\hline & & & جنسييت \\
\hline$\varphi$ & ir & نهتر & \\
\hline r & \&N & سطع | & \\
\hline 9 & ru & ra ra & سطح شدت أُتيسم \\
\hline 1 & r & سطج " & \\
\hline
\end{tabular}

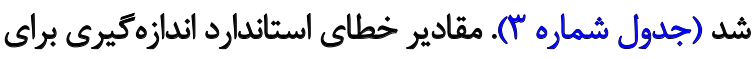

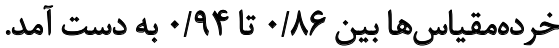

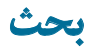

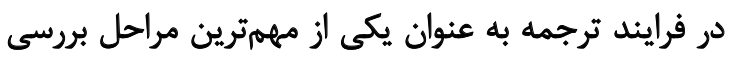

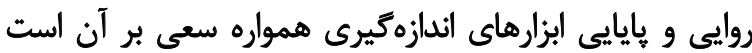

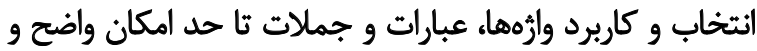

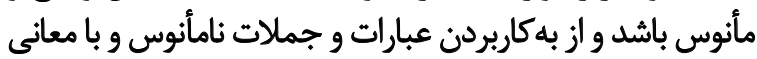

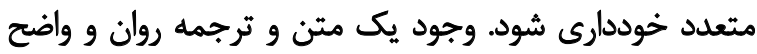

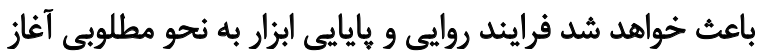

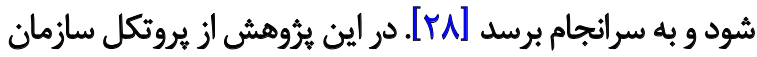

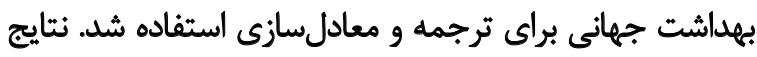

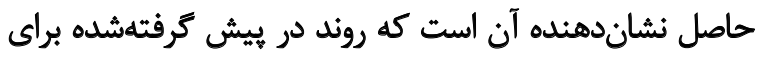

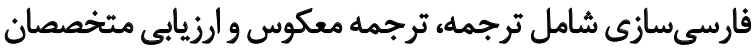

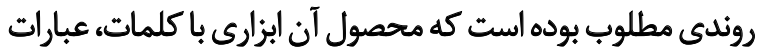
و جملات صحيح، واضح و شفاف بوده است.

بس از تهيه نسخه فارسى نهايى يرسشنامهن، روايى ابزار

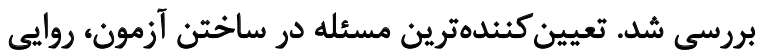

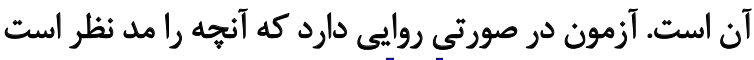

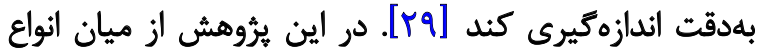

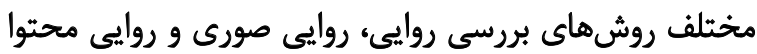

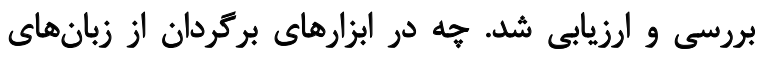

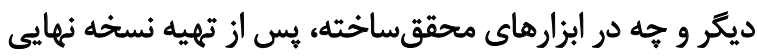

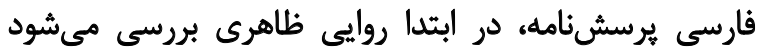

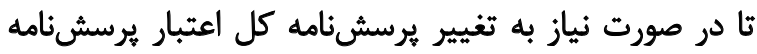

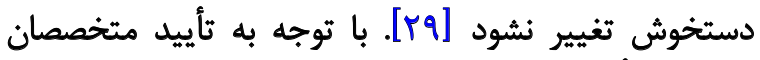

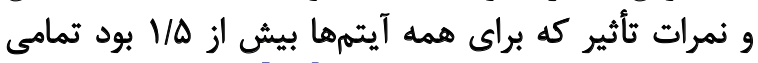

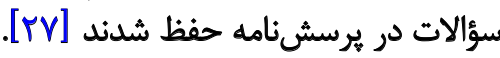

نتايج حاصل از تحليل اطلاعات به منظور بررسى روايى محتوا نشاندهنده آن بود كه مقدار ضريب نسبى روايى محتوا براى بلى روائ
عبارات به لحاظ وضوح و كاربرد زبان مشترى بردي برى و نأييد

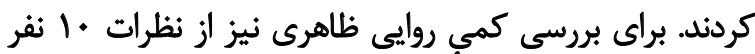

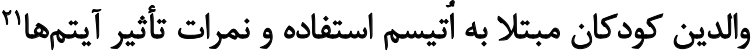

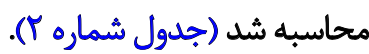

به منظور بررسى روايي محتواييى از • ا نفر متخصص كاردرمانى

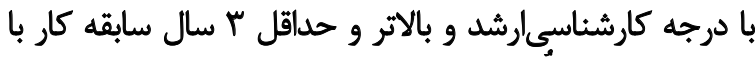

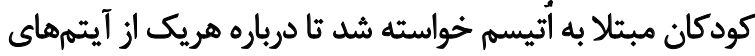

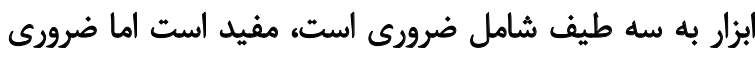

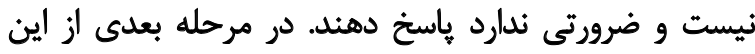

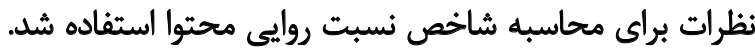

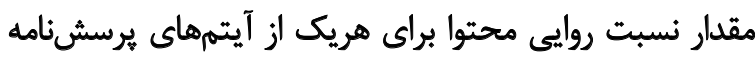

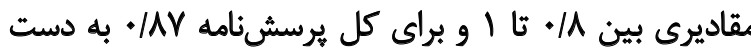

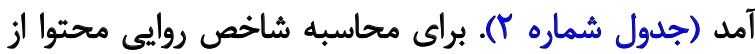

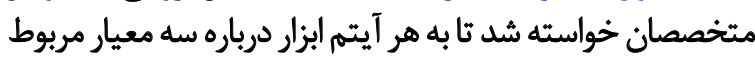

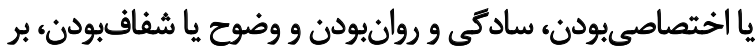

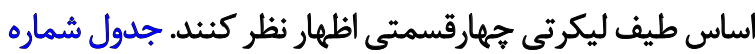

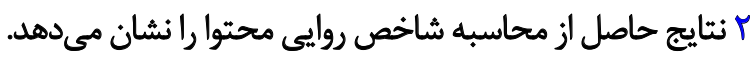

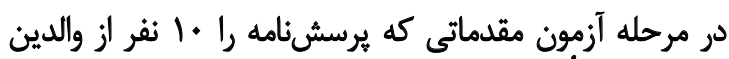

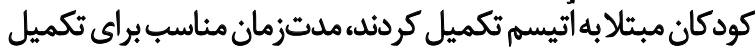

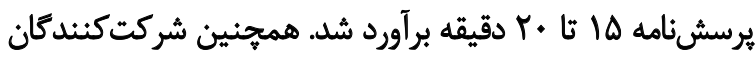

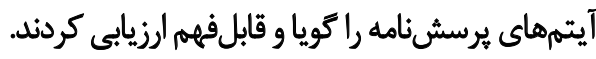
براى بررسى ثبات درونى كل يرسشنامه و خردهمقياسهاى

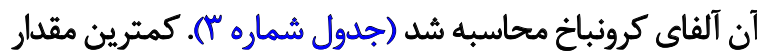

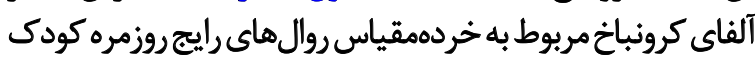

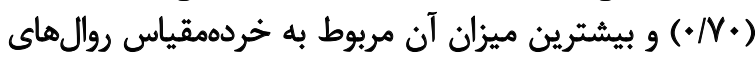

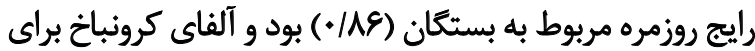

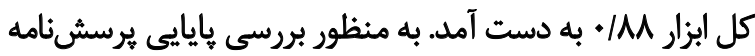

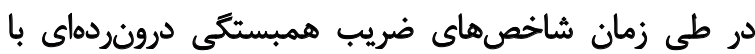

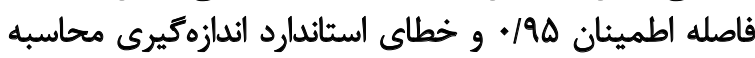

21. Item impact scores 
جدول Y. نتايج بروسى روائي برسشثامه با شاخص نمره ثأثير آيتمها، CVI , CVR

\begin{tabular}{|c|c|c|c|}
\hline CVI & CVR & نمره تأثير & آيتيم \\
\hline 1 & 1 & $1 / 8$ & 1 \\
\hline 1 & 1 & $I / V$ & $r$ \\
\hline س9/. & 1 & $1 / 80$ & $r$ \\
\hline 1 & 1 & V/FA & r \\
\hline ./AM &.$/ 1$ & $V / F$ & $\Delta$ \\
\hline "צr/. & .11 & INA & 8 \\
\hline 1 & $\cdot / 1$ & $V / F$ & $\gamma$ \\
\hline 1 &.$/ 1$ & $V / \Delta \Delta$ & $A$ \\
\hline 1 & 1 & INA & 9 \\
\hline 1 & 1 & $\mathrm{I} / \mathrm{V}$ & 1. \\
\hline 1 &.$/ 1$ & INA & 11 \\
\hline r & 1 & $T / N E$ & ir \\
\hline.$/ Q$ & .11 & V/EA & ir \\
\hline 1 & 1 & $T / \Delta S$ & if \\
\hline 1 & 1 & $\mathrm{~V} / \mathrm{V}$ & 10 \\
\hline 1 & $\cdot / 1$ & $1 / 8$ & 18 \\
\hline 1 & $\cdot / 1$ & IND & iv \\
\hline 1 &.$/ 1$ & $T / 1$ & in \\
\hline.$/ Q$ &.$/ 1$ & $r / . r$ & 19 \\
\hline 1 & 1 & T/Q9 & r. \\
\hline 1 & $\cdot / 1$ & $r / \Delta Q$ & $M$ \\
\hline 1 & 1 & r/TA & $r$ \\
\hline.$/ 9$ & $\cdot / 1$ & $\mathbb{N A}$ & r \\
\hline 1 & .11 & $r / 1$ & $r$ \\
\hline 1 & $\cdot / 1$ & $r / \Lambda$ & ro \\
\hline 1 & 1 & $r / 1$ & re \\
\hline . &.$/ A$ & $r / F$ & rv \\
\hline.$/ Q$ & $\cdot / 1$ & $\mathrm{~V} / \mathrm{V}$ & YA \\
\hline.$/ Q$ &.$/ 1$ & $\mathrm{I} / \mathrm{V}$ & rq \\
\hline 1 & .11 & $r / * F$ & r. \\
\hline 1 & .11 & $\mathrm{I} / \mathrm{V}$ & $m$ \\
\hline 1 &.$/ 1$ & $V / \Delta \Delta$ & $\pi$ \\
\hline.$/ 97$ & $\cdot / A V$ & & هيانكين كل \\
\hline
\end{tabular}




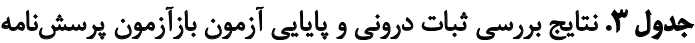

\begin{tabular}{|c|c|c|c|c|}
\hline خطاى استأدارد اندازهيكيرى (SEM) & فاصله|طمينان & ضريب همبستكى درونردهاى (ICC) & ألفاى كرونباخ & خرده مقياس \\
\hline.$/ 91$ & $. / A Y=9 Y$ &.$/ 94$ &.$M$ & باهميودن والدين و فرزندان \\
\hline$\cdot M$ & $. / 19-94$ &.$/ 94$ & $\cdot M F$ & باهميودن زوجين \\
\hline$\cdot M T$ & $\cdot / \lambda Y-q Y$ & . & $* N$ & روالهاى رايج روزمره كودى \\
\hline.$/ 4 r$ & $\cdot / A Y-q \vee$ &.$/ 94$ & . ART & باهميودن در وعلدهاي غذايى \\
\hline$\cdot M$ & $\cdot / M-9 Y$ &.$/ 94$ &.$M r$ & زمان باهميودن خانواده \\
\hline . Iar & $\cdot / M^{4}-q$ & $\cdot \mathbb{N}$ & $\cdot M$ & روال هاى رائج مربوط به كارهاى خانه \\
\hline $.18 A$ & /AY_qY & ./94 & $+\operatorname{lN}$ & روالهاى رايج مربوط به بستكان \\
\hline$y \cdot r$ & $\cdot / M^{*}-94$ & . /AY & $\cdot M r$ & روالهاى رايج مربوط به مديريت خانواده \\
\hline$Y M$ & $. / 91-94$ &.$/ 9$ & $\mathscr{I M}$ & نمره كل \\
\hline
\end{tabular}

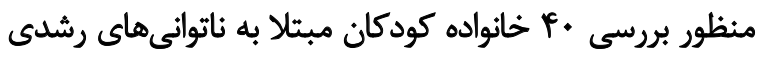

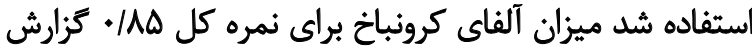

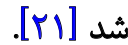

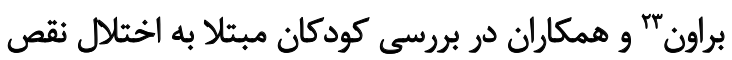

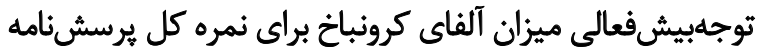

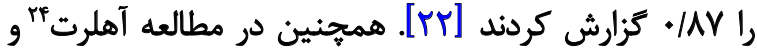

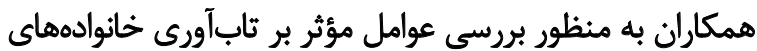

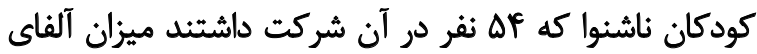

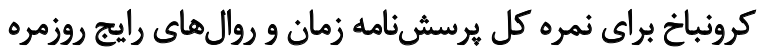

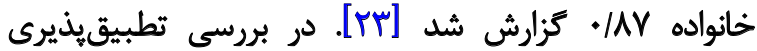

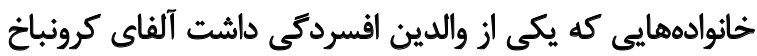

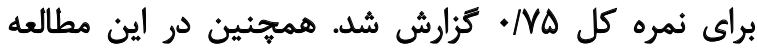

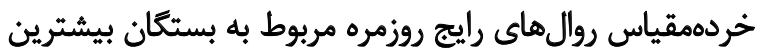

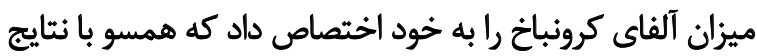

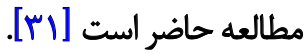

\section{نتيجلمكيرى}

نسخه فارسى يرسشنامه زمان و روالهاى رايج روزمره خانواده

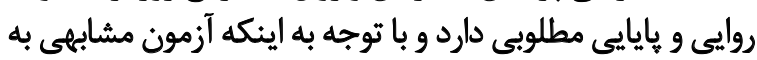

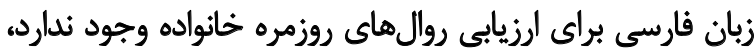

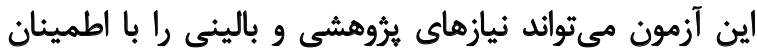

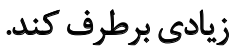

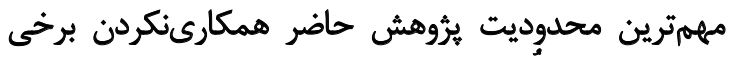

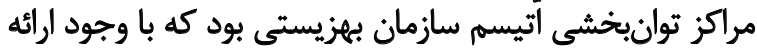

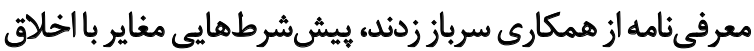

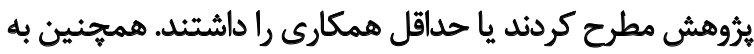
دليل دسترسىنداشتن به ابزارهاى مشابه در كشور امكان بررسى إنى

23. Brown

24. Ahlert

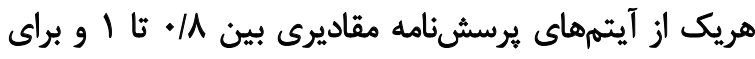

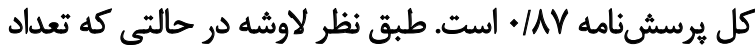

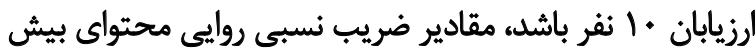

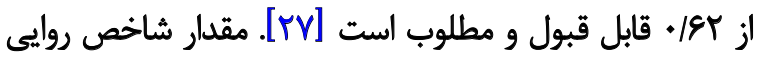

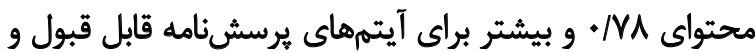

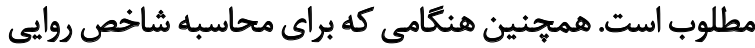

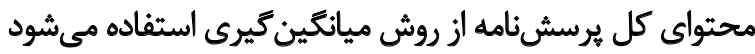

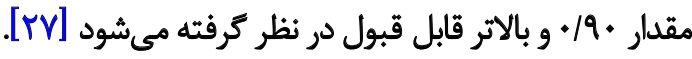
يايايى شاخصى براي ارزشيابى آزمونها و برسشنامهنها اعم

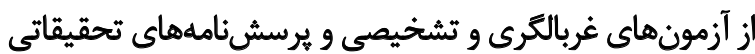

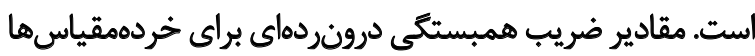

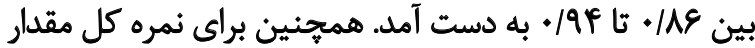

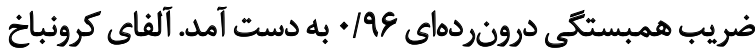

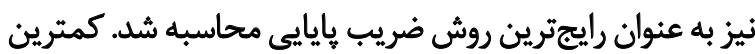

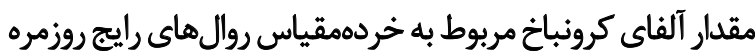

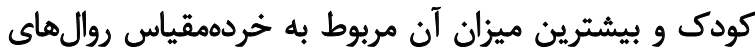

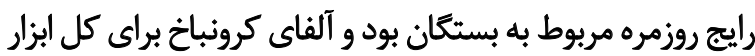

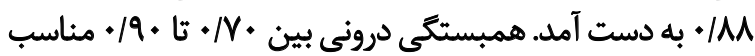

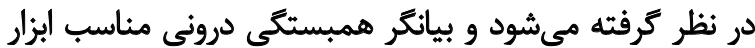

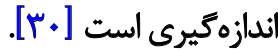

نتايج مطالعه حاضر با نتايج مطالعات كذشته كه مقدار آلفاى

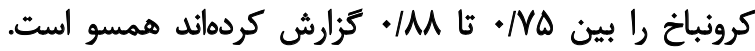

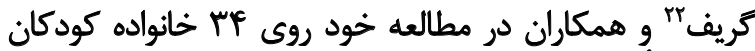

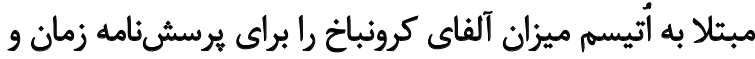

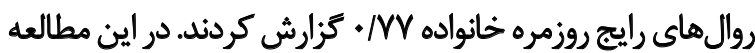

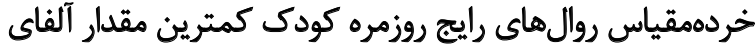

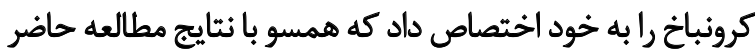

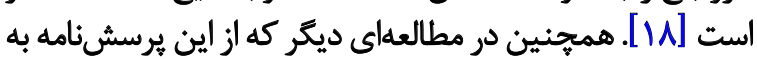


روايى همزمان وجود نداشت. به علت تنوع سبك زندگى در مناطق مختلف كشور بررسى و مقايسه روايى محتوايى در مناطق ندان و قوميتهاى مختلف ييشنهاد مىشود.

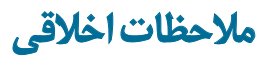
يبروى أز اصول الخلاق يثوهش اصول اخلاقى ثروهش براى اين مطالعه به تصويب كميته

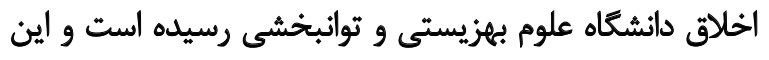

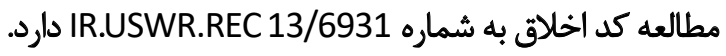

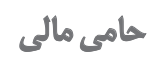

مقاله حاضر از ياياننامه كارشناسى ارشد احسان جمشيديان از

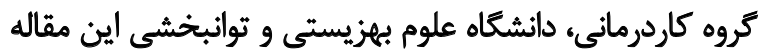
استخراج شده است. مشاركت نويسندكًان

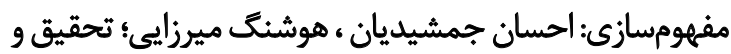

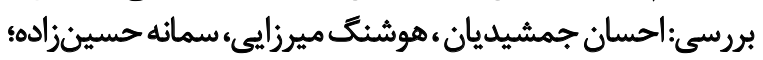

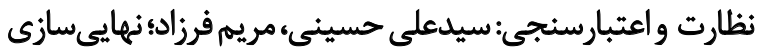
نوشته: احسان جمشيديان.

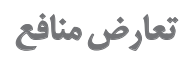

بنابر اظهار نويسندكان، اين مقاله تعارض منافع ندارد. 


\section{References}

[1] Baio J. Developmental disabilities monitoring network surveillance year 2010 principal investigators Centers for Disease Control and prevention (CDC). Prevalence of autism spectrum disorder among children aged 8 years-Autism and developmental disabilities monitoring network, 11 sites, United States, 2010. MMWR Surveill Summaries. 2014; 63(2):1-21. [PMID]

[2] American Psychiatric Association. Diagnostic and Statistical Manual of Mental Disorders (DSM- $\left.{ }^{\circledR}\right)$. Washington: American Psychiatric Publication; 2013. [DOI:10.1176/appi. books.9780890425596]

[3] Cosbey J, Johnston SS, Dunn ML. Sensory processing disorders and social participation. American Journal of Occupational Therapy. 2010; 64(3):462-73. [DOI:10.5014/ajot.2010.09076] [PMID]

[4] Shiri V, Hosseini SA, Pishyareh E, Nejati V, Biglarian A. [Study the relationship of executive functions with behavioral symptoms in children with high-functioning autism (Persian)]. Archives of Rehabilitation. 2015; 16(3):208-17.

[5] Bahramkhani M, Darvishi N, Keshavarz Z, Dadkhah A. [The camparison of executive functions in normal and autistic children, considering mathematics and reading abilities (Persian)]. Journal of Rehabilitation. 2013; 13(Special Issue):128-35.

[6] Davarinia A, Yarmohammadian A, Ghamarani A. The comparave study of gross and fine motor skills and body balance in children with intellectual disability, ausm and learning disorder with normal children. Archives of Rehabilitation. 2015; 16(1): 66-75.

[7] Bagby MS, Dickie VA, Baranek GT. How sensory experiences of children with and without autism affect family occupations. American Journal of Occupational Therapy. 2012; 66(1):78-86. [DOI:10.5014/ajot.2012.000604] [PMID]

[8] Schaaf RC, Toth-Cohen S, Johnson SL, Outten G, Benevides TW. The everyday routines of families of children with autism: Examining the impact of sensory processing difficulties on the family. Autism. 2011; 15(3):373-89. [DOI:10.1177/1362361310386505] [PMID]

[9] Mohammadpour M, Rassafiani M, Ahmadi KM, Behnia F, Haghgoo HA, Biglarian A. [Comparing the time-use of mothers with autistic children with that of mothers with healthy ones (Persian)]. Journal of Research in Rehabilitation Sciences. 2014; 10(1):182-92.

[10] Amini DA, Kannenberg K, Bodison S, Chang P, Colaianni D, Goodrich B, et al. Occupational therapy practice framework: Domain \& process 3rd edition. American Journal of Occupational Therapy. 2014; 68(Supple 1):S1-S48. [DOI:10.5014/ ajot.2014.682006]

[11] Boyd BA, McCarty CH, Sethi C. Families of children with autism: A synthesis of family routines literature. Journal of Occupational Science. 2014; 21(3):322-33. [DOI:10.1080/144275 91.2014.908816]

[12] Brown O, Fouche P, Coetzee M. Bouncing forward: Families living with a type I diabetic child. South African Family Practice. 2010; 52(6):536-41. [DOI:10.1080/20786204.2010.10874044]
[13] Rodger S, Umaibalan V. The routines and rituals of families of typically developing children compared with families of children with autism spectrum disorder: An exploratory study. British Journal of Occupational Therapy. 2011; 74(1):20-6. [DO I:10.4276/030802211X12947686093567]

[14] Spagnola M, Fiese BH. Family routines and rituals: A context for development in the lives of young children. Infants \& Young Children. 2007; 20(4):284-99. [DOI:10.1097/01. IYC.0000290352.32170.5a]

[15] Behnia F, Rassafiani M, Nakhai S, Mohammadpour M, Ahmadi KM. Time use of mothers of children with an autism spectrum disorder: A comparative study. Iranian Rehabilitation Journal. 2017; 15(1):49-56. [DOI:10.18869/nrip.irj.15.1.49]

[16] Hoogsteen L, Woodgate RL. Centering autism within the family: A qualitative approach to autism and the family. Journal of Pediatric Nursing. 2013; 28(2):135-40. [DOI:10.1016/j. pedn.2012.06.002] [PMID]

[17] Fiese BH, Tomcho TJ, Douglas M, Josephs K, Poltrock S, Baker T. A review of 50 years of research on naturally occurring family routines and rituals: Cause for celebration? Journal of Family Psychology. 2002; 16(4):381-390. [DOI:10.1037/08933200.16.4.381] [PMID]

[18] Greeff AP, Van der Walt KJ. Resilience in families with an autistic child. Education and Training in Autism and Developmental Disabilities. 2010; 45(3):347-55.

[19] Jensen EW, James SA, Boyce WT, Hartnett SA. The family routines inventory: Development and validation. Social Science \& Medicine. 1983; 17(4):201-11. [DOI:10.1016/02779536(83)90117-X]

[20] McCubbin H, McCubbin M, Thompson Al. Family time and routines index. Family assessment inventories for research and practice. Madison: University of Wisconsin-Madison; 1987.

[21] Greeff AP, Nolting C. Resilience in families of children with developmental disabilities. Families, Systems, \& Health. 2013; 31(4):396-405. [DOI:10.1037/a0035059] [PMID]

[22] Brown O, Howcroft G, Muthen T. Resilience in families liv ing with a child diagnosed with hyperactivity/attention deficit disorder. South African Journal of Psychology. 2010; 40(3):33850. [DOI:10.1177/008124631004000312]

[23] Ahlert IA, Greeff AP. Resilience factors associated with adaptation in families with deaf and hard of hearing children. American Annals of the Deaf. 2012; 157(4):391-404. [DOI:10.1353/ aad.2012.1629]

[24] Harakraj N. Resilience in Indian families in which a member has died [MSc. thesis]. KwaDlangezwa: University of Zululand; 2006.

[25] Abdul Jalal FH. Family functioning and adolescent delinquency in Malaysia [PhD dissertation]. lowa: lowa State University; 2005.

[26] McCubbin HI, Thompson Al. Family assessment inventories for research and practice. Madison, Wisconsin: University of Wisconsin Publishers; 1987. 
[27] Taghizadeh Z, Ebadi A, Montazeri A, Shahvari Z, Tavousi M, Bagherzadeh R. [Psychometric properties of health related measures. Part 1: Translation, development, and content and face validity (Persian)]. Payesh. 2017; 16(3):343-57.

[28] Kasehchi M, Behnia F, Mirzaee H, Rasafyani M, Farzi M, Gharib M. [Validity and reliability of Persian version of highfunctioning autism spectrum screening questionnaire age 7-12 (Persian)]. Pajouhan Scientific Journal. 2013; 12(1):45-54.

[29] Mohammadbeigi A, Mohammadsalehi N, Aligol M. [Validity and reliability of the instruments and types of measurments in health applied researches (Persian)]. Journal of Rafsanjan University of Medical Sciences. 2015; 13(12):1153-70.

[30] Ebadi A, Taghizadeh Z, Montazeri A, Shahvari Z, Tavousi M, Bagherzadeh R. [Translation, development and psychometric properties of health related measures-part 2: Construct validity, reliability and responsiveness (Persian)]. Payesh. 2017; 16(4):445-55.

[31] Bester C. The identification of resilience in, and the development of a corresponding intervention programme for families with a parent living with major depressive disorder [PhD dissertation]. Stellenbosch: University of Stellenbosch; 2009. 


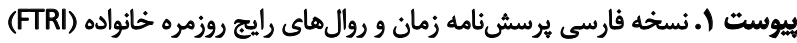

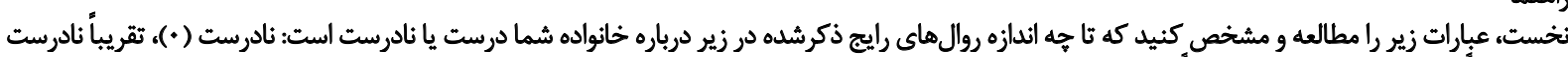

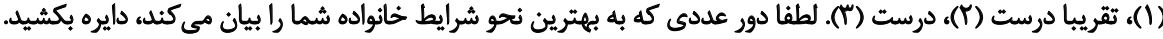

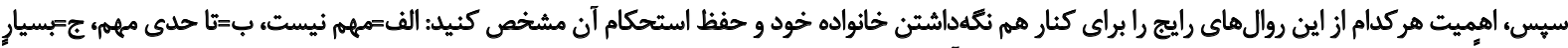

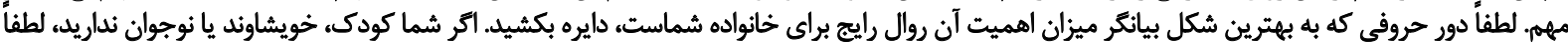

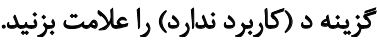

\begin{tabular}{|c|c|c|c|c|c|c|c|c|}
\hline \multicolumn{4}{|c|}{ ميزان اهميت در استحكام خانواده } & \multirow{3}{*}{ درست } & \multirow{3}{*}{ تقريباً درست } & \multirow{3}{*}{ نادرسيث } & \multirow{3}{*}{ نادرست } & روالهاي رايج \\
\hline \multirow{2}{*}{ كاربرد } & \multicolumn{3}{|c|}{ اهميث براي خانواده } & & & & & \multirow{2}{*}{ روالهاي رايج روزهاي كارى و اوقات } \\
\hline & مهيلى & تا حدى & مهير نيست & & & & & \\
\hline 2 & ₹ & ب & الف & $r$ & r & 1 & . & 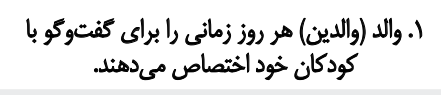 \\
\hline$د$ & $\varepsilon$ & ب & الف & $r$ & $r$ & 1 & - & 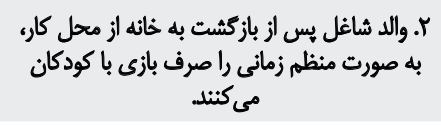 \\
\hline 2 & $\varepsilon$ & ب & الف & $r$ & r & 1 & - & ب. والد شاغل تثريياً هر روز زمانى رال صرف مراقبت \\
\hline 2 & ๕ & ب & الف & $r$ & r & 1 & - & 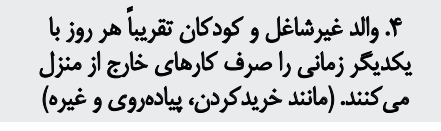 \\
\hline 2 & ₹ & ب & الف & $r$ & r & 1 & • & 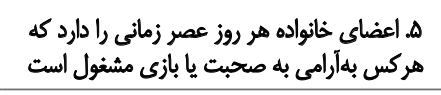 \\
\hline \multicolumn{4}{|c|}{ ميزان اهميت در استحكام خانواده } & \multirow{2}{*}{ درست } & \multirow{2}{*}{ تقريباً درست } & \multirow{2}{*}{ 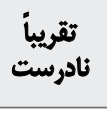 } & \multirow{2}{*}{ 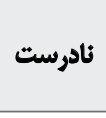 } & \multirow{2}{*}{ روالهاى رايج } \\
\hline كاربرد ندارد & & كخاثواده & اهميث ؛ & & & & & \\
\hline 2 & ๕ & ب & الف & $r$ & $r$ & 1 & • & 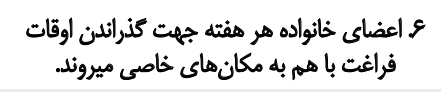 \\
\hline 2 & $\varepsilon$ & ب & الف & $r$ & r & 1 & • & 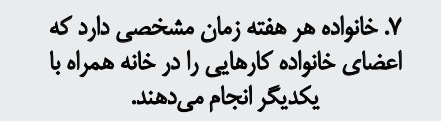 \\
\hline 2 & ₹ & ب & الف & $r$ & $r$ & 1 & • & ه والد (والدين) تقريباً هر روز براي كودكان داستان \\
\hline 2 & ๕ & ب & الف & $r$ & $r$ & 1 & • & 9. هر كودى هـ روز زمانى برائ تثها بازى كردن \\
\hline 2 & ๕ & ب & الف & $r$ & r & 1 & . & . . كودكان / ثوجوانان هر روز با دوستان خود \\
\hline 2 & $\varepsilon$ & ب & الف & $r$ & r & 1 & - & 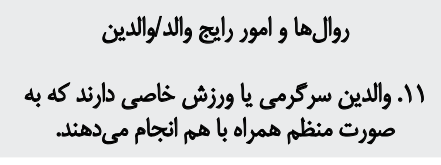 \\
\hline$د$ & $\varepsilon$ & ب & الف & $r$ & $r$ & 1 & - & با. والدين اغلبه زمانى را با يكديكر سيرى \\
\hline 2 & ? & ب & الف & $r$ & r & 1 & - & זا. واللاين يك يا جند بار در هفته با هم ييرون \\
\hline 2 & ? & ب & الف & $r$ & $r$ & 1 & $\cdot$ & 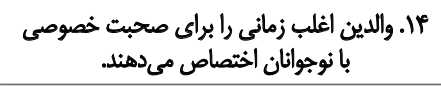 \\
\hline
\end{tabular}




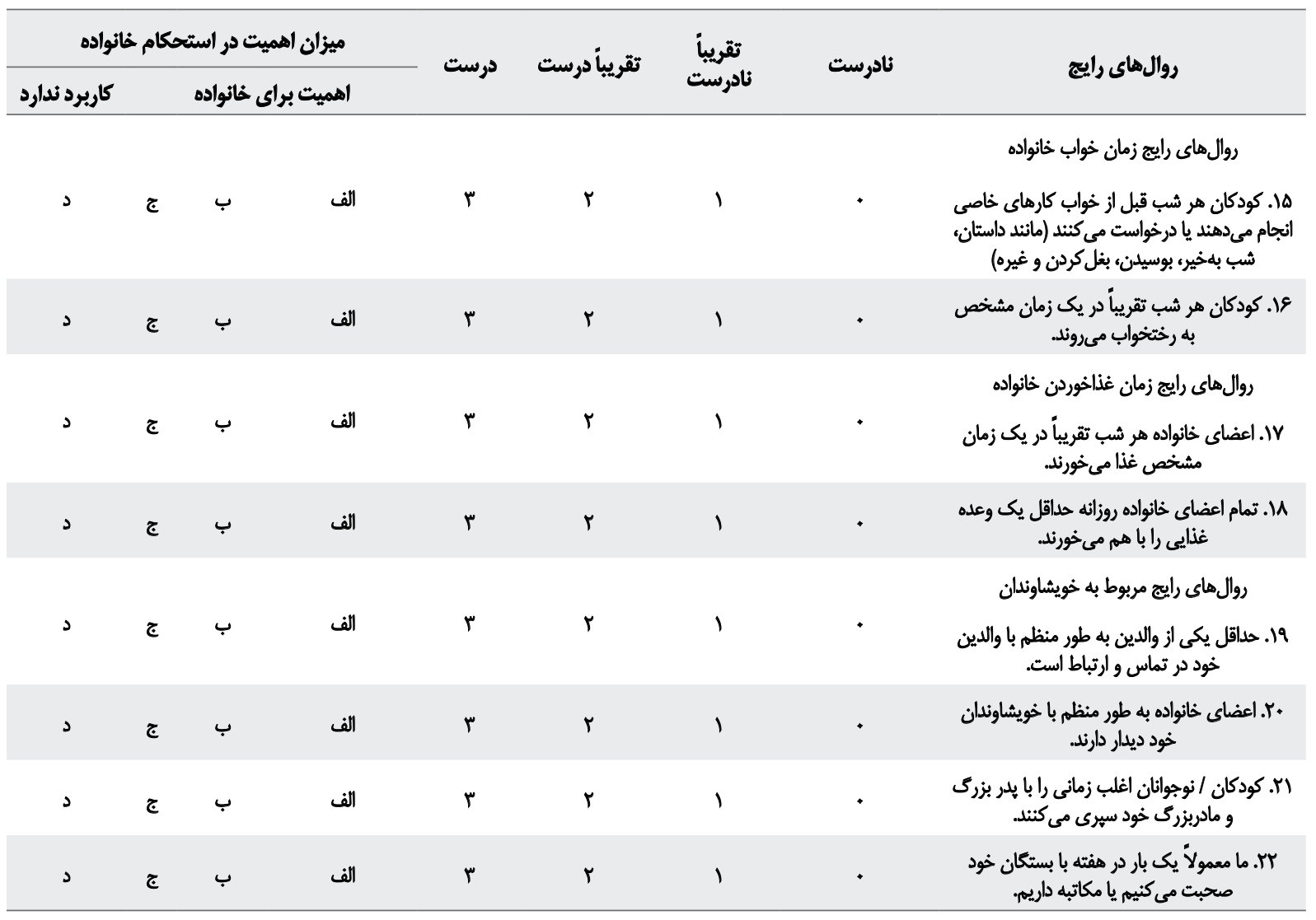

\begin{tabular}{|c|c|c|c|c|c|c|c|c|}
\hline \multicolumn{4}{|c|}{ ميزان الهميت در استحكام خانواده } & \multirow{2}{*}{ 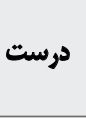 } & \multirow{2}{*}{ تقريباً درست } & \multirow{2}{*}{ ت تادرسيباً } & \multirow{2}{*}{ نادرست } & \multirow{2}{*}{ روالهاي رايج } \\
\hline كاربرد ندارد & & فانواده & الهمي & & & & & \\
\hline 2 & ⿷ & 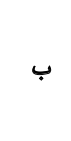 & الف الف & $r$ & $r$ & 1 & • & 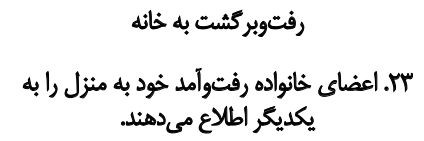 \\
\hline 2 & $\varepsilon$ & ب & الف & $r$ & r & 1 & - & 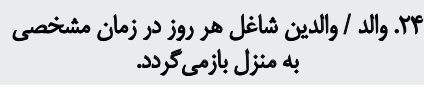 \\
\hline 2 & $\varepsilon$ & ب & الف - الف & $r$ & r & 1 & - & 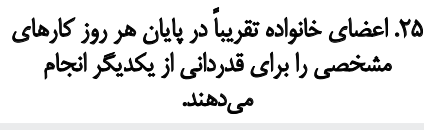 \\
\hline 2 & $\varepsilon$ & 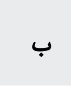 & الف & r & $r$ & 1 & - & عז. ما هر روز توجان و محدبث خود را به يكديكر \\
\hline$د$ & 飞 & ب & الف & r & $r$ & 1 & • & 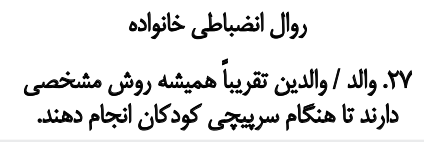 \\
\hline$د$ & ๕ & ب & الف & $r$ & $r$ & 1 & • & 1اك. والدين اغلب درباره قوانين جديد باكودكان/ \\
\hline & & & & & & & & امور خاثه \\
\hline$s$ & ๕ & 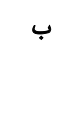 & الف - ت الف & $r$ & r & 1 & - & 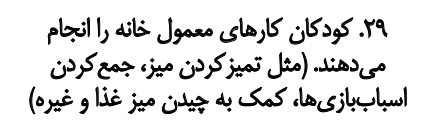 \\
\hline$د$ & ๕ & 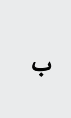 & الف & $r$ & $r$ & 1 & • & 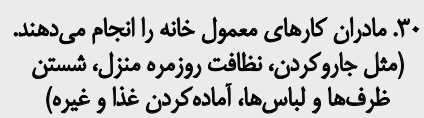 \\
\hline
\end{tabular}




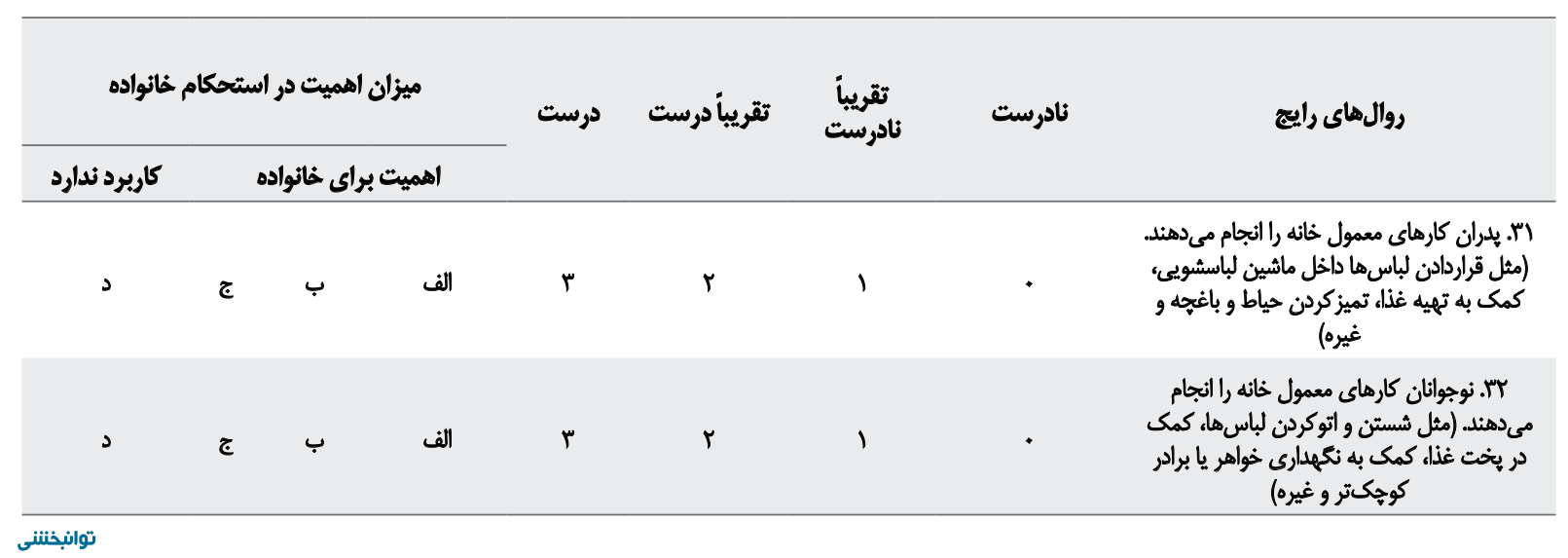


This Page Intentionally Left Blank 